\title{
Diabetic Foot Ulcer with Alcaligenes faecalis Infection
}

\author{
Chienhsiu Huang \\ Department of Internal Medicine, Dalin Tzu Chi Hospital, Dalin Town, Taiwan
}

\author{
Keywords \\ Alcaligenes faecalis · Diabetic foot ulcer · Extensive drug \\ resistant
}

\begin{abstract}
Background: Diabetic foot ulcers are an increasingly common complex problem and are associated with a very considerable health care burden. Diabetic foot ulcer with Alcaligenes faecalis infection is rarely reported in the literature. We report a case series of diabetic foot ulcer with $A$. faecalis infection treated at our facility. Methods: We conducted a retrospective analysis of all patients with diabetic foot ulcer with A. faecalis infection seen from January 2014 to April 2019. We analyzed the clinical characteristics, ulcer lesion classification, comorbidities, prior intravenous antibiotic use within 3 months, wound culture, antibiotics sensitivity test, and clinical outcomes of these patients. Results: Eight cases of diabetic foot ulcer with $A$. faecalis infection were seen in 5 males and 3 females. Mean age was 54.6 years. All patients had other comorbidities, and all ulcer lesions were of chronic duration ( $>14$ days). All wound cultures revealed polymicrobial infection, with 2 cases of diabetic foot with extensive drug-resistant $A$. faecalis infection found in 2019. All patients needed intravenous antibiotic therapy and surgical interventions for the chronic ulcer lesion. The wound failed to heal in 3 patients. Conclusions: All diabetic foot ulcers with A. faecalis infection were of chronic duration ( $>14$ days) and had polymicrobial infection. Extensive drug-resistant $A$. fae-
\end{abstract}

karger@karger.com www.karger.com/dde

Karger $\stackrel{\text { ' }}{=}$

BOPEN ACCESS
(C) 2020 The Author(s)

Published by S. Karger AG, Basel

This article is licensed under the Creative Commons AttributionNonCommercial-NoDerivatives 4.0 International License (CC BYNC-ND) (http://www.karger.com/Services/OpenAccessLicense) Usage and distribution for commercial purposes as well as any distribution of modified material requires written permission. calis emerged in 2019. Definitive antibiotic therapy is necessary for all infected wounds and should be based on both the culture results and susceptibility data. All patients will need appropriate wound care, and most will need rapid surgical intervention for an optimal outcome.

(C) 2020 The Author(s)

Published by S. Karger AG, Basel

\section{Introduction}

Diabetic foot ulcers are an increasingly common complex problem and are associated with a very considerable health care burden [1]. Foot ulcer is the most frequent diabetes-related cause of hospitalization. The global prevalence of diabetic foot ulcer is 6.3\% [2]. People with diabetes have an about $25 \%$ chance of developing a foot ulcer in their lifetime [3]. About half of all diabetic foot ulcers are infected at presentation [4]. Acute infections in patients who have not recently received antimicrobials are often monomicrobial. Gram-positive cocci are the predominant organisms, and Staphylococcus aureus is the most commonly isolated pathogen. Chronic infections are often polymicrobial, including Enterococci, Enterobacteriaceae, Pseudomonas aeruginosa, other non-fermenting Gram-negative bacilli, and obligate anaerobes [5-9].

Alcaligenes faecalis is a Gram-negative, obligate aerobe, oxidase-positive, catalase-positive, and non-fermenting bacterium commonly found in soil, water, and hospital environments. The organism has been isolated from a range 
Table 1. Clinical characteristics and treatment outcome in 8 cases of diabetic foot ulcer with Alcaligenes faecalis infection

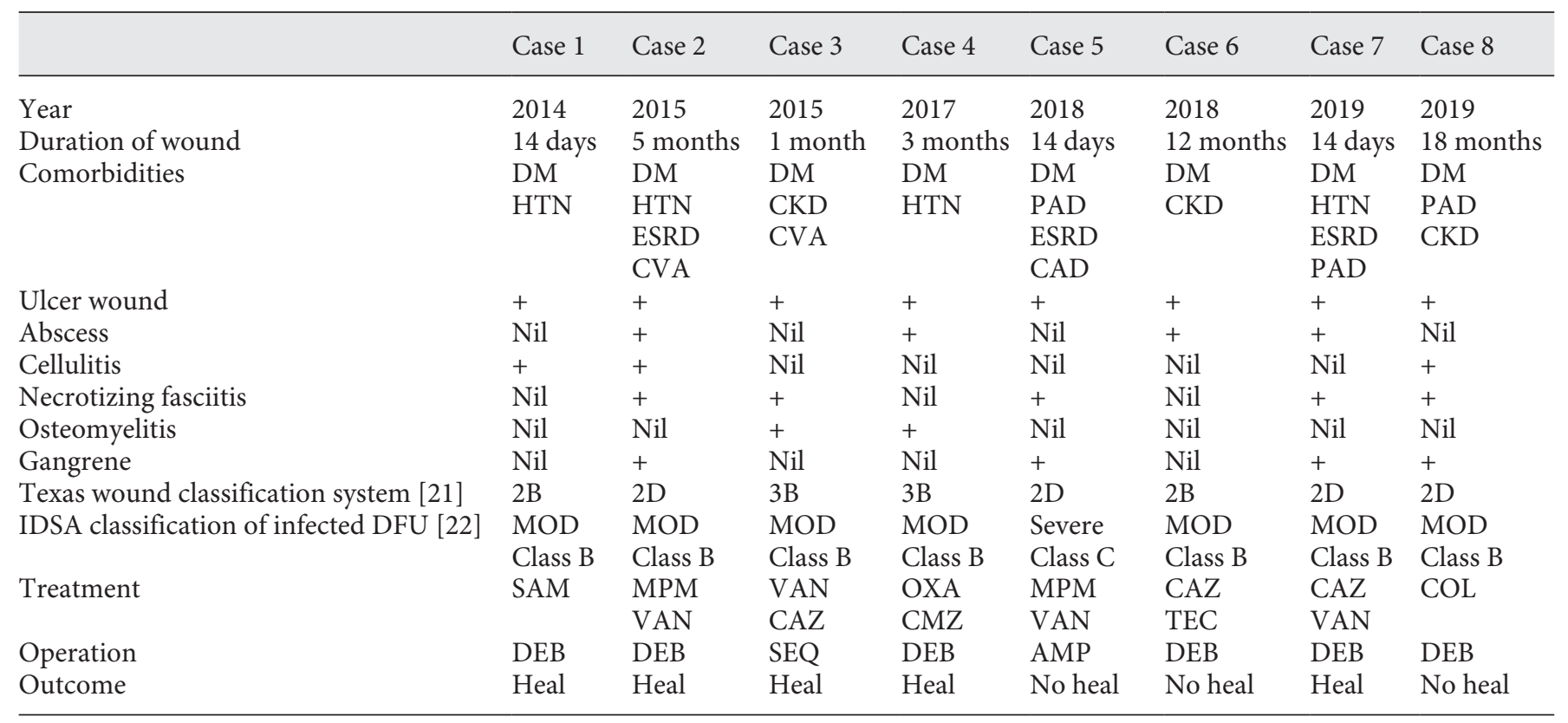

DM, diabetes mellitus; HTN, hypertension; ESRD, end-stage renal disease; CVA, cerebral vascular accident; CKD, chronic kidney disease; PAD, peripheral artery disease; CAD; coronary artery disease.

IDSA, Infectious Disease Society of America; DFU, diabetic foot ulcer; MOD, moderate.

SAM, ampicillin-sulbactam; MPM, meropenem; VAN, vancomycin; CAZ, ceftazidime; OXA, oxacillin; CMZ, cefmetazole; TEC, teicoplanin; COL, colistin.

DEB, debridement; SEQ, sequestrectomy; AMP, amputation.

of clinical materials such as urine, blood, wound discharge, stool, cerebrospinal fluid, and respiratory secretions [1012]. A. faecalis has been associated with endocarditis, bacteremia, meningitis, endophthalmitis, skin and soft tissue infection, urinary tract infection, otitis media, peritonitis, and pneumonia [13-20]. We here report a series of patients with diabetic foot ulcer with $A$. faecalis infection, in order to improve the clinical care of such patients.

\section{Methods}

We conducted a retrospective analysis of all patients presenting with diabetic foot ulcer with $A$. faecalis infection who were admitted to Dalin Tzu Chi Hospital, Taiwan, from January 2014 through April 2019. Patients were diagnosed with diabetic foot ulcer with infection when their clinical symptoms and signs indicated skin and soft tissue infection, such as redness, warmth, induration, tenderness, ulcer lesion, abscess, gangrene, fasciitis, or osteomyelitis. Ulcer lesion classification was done by using the University of Texas wound classification system [21], and the infected diabetic foot ulcer classification system of the Infectious Disease Society of America [22]. The medical records of all patients were reviewed for demographic information, clinical symptoms and signs, pharmacological therapy for blood sugar, admission HbA1c values, blood sugar val- ues (at 6 a.m., 11 a.m., 5 p.m., and 9 p.m.), comorbidities, use of intravenous (IV) antibiotics within 3 months, wound culture, antibiotics sensitivity test, and clinical outcomes. Superficial wound swab specimens were collected for culture. Specimens were obtained only after the wound was cleaned using povidone iodine solution and sterile saline. Wound swab specimens for antibiotic susceptibility were done using the Vitek II system with ASTGN87 cards (BioMérieux, Marcy-l'Étoile, France) with the interpretive criteria M10025 th of the Clinical and Laboratory Standards Institute.

Wound swab specimens were cultured as soon as they were collected, on chocolate agar, blood agar, eosin methylene blue agar, colistin-nalidixic acid agar plates, and thioglycollate broth. Gram staining and microscopy were prepared and read. The 5 culture mediums were incubated in a carbon dioxide-enriched atmosphere for 16$18 \mathrm{~h}$. Then, a sterile swab stick was used to transfer a sufficient number of colonies of pure culture and to suspend the microorganisms in $3.0 \mathrm{~mL}$ of sterile saline (aqueous $0.45-0.50 \% \mathrm{NaCl}, \mathrm{pH} 4.5-7.0$ ) in a $12 \times 75 \mathrm{~mm}$ clear plastic test tube. The turbidity was adjusted to 0.50-0.63 McFarland Equivalence Turbidity Standard. Identification cards were inoculated with microorganism suspensions using an integrated vacuum apparatus. A test tube containing the microorganism suspension was placed into a cassette. One GN identification card (automated identification of 135 taxa of the most significant fermenting and non-fermenting Gram-negative bacilli) and another VITEK II AST-N322 card (for susceptibility testing of aerobic Gramnegative bacilli against specified antimicrobials) were placed in the 
Table 2. Bacteriology in 8 cases of diabetic foot ulcer with Alcaligenes faecalis infection

\begin{tabular}{|c|c|c|c|c|c|c|c|c|}
\hline & Case 1 & Case 2 & Case 3 & Case 4 & Case 5 & Case 6 & Case 7 & Case 8 \\
\hline Year & 2014 & 2015 & 2015 & 2017 & 2018 & 2018 & 2019 & 2019 \\
\hline \multirow{4}{*}{$\begin{array}{l}\text { Prior intravenous antibiotics used } \\
\text { within } 3 \text { months }\end{array}$} & CIP & PIP & Nil & CEF & Nil & CAZ & CAZ & LVX \\
\hline & SAM & $\mathrm{CRO}$ & & TEC & & CIP & VAN & MPM \\
\hline & & VA & & & & PIP & & TEC \\
\hline & & & & & & TEC & & \\
\hline \multicolumn{9}{|c|}{ Results of antibiotics sensitivity test of Alcaligenes faecalis } \\
\hline GM & S & $\mathrm{S}$ & S & $\mathrm{R}$ & $\mathrm{S}$ & I & $\mathrm{R}$ & $\mathrm{R}$ \\
\hline AN & $\mathrm{S}$ & $\mathrm{S}$ & $\mathrm{S}$ & $\mathrm{S}$ & $\mathrm{S}$ & $\mathrm{S}$ & $\mathrm{R}$ & $\mathrm{R}$ \\
\hline CAZ & $\mathrm{S}$ & $\mathrm{S}$ & S & $\mathrm{s}$ & $\mathrm{S}$ & $\mathrm{s}$ & $\mathrm{R}$ & $\mathrm{R}$ \\
\hline FEP & S & S & S & I & S & I & $\mathrm{R}$ & $\mathrm{R}$ \\
\hline SAM & $\mathrm{S}$ & S & S & I & S & S & $\mathrm{R}$ & $\mathrm{R}$ \\
\hline TZP & $\mathrm{S}$ & S & $\mathrm{S}$ & I & $\mathrm{S}$ & $\mathrm{S}$ & $\mathrm{R}$ & $\mathrm{R}$ \\
\hline CIP & S & S & S & $\mathrm{R}$ & S & $\mathrm{R}$ & $\mathrm{R}$ & $\mathrm{R}$ \\
\hline IPM & S & S & S & S & S & S & $\mathrm{R}$ & $\mathrm{R}$ \\
\hline MPM & S & S & $\mathrm{S}$ & $\mathrm{S}$ & $\mathrm{S}$ & $\mathrm{S}$ & $\mathrm{R}$ & $\mathrm{R}$ \\
\hline TGC & NA & NA & NA & NA & NA & NA & NA & S \\
\hline \multicolumn{9}{|l|}{ Mixed infection pathogens } \\
\hline Enterococcus & $\mathrm{V}$ & & & & $\mathrm{V}$ & & & V \\
\hline Proteus vulgaris & & $\mathrm{V}$ & & & $\mathrm{V}$ & & & \\
\hline Citrobacter koseri & & & V & & & & & \\
\hline MRSA & & & V & & & & & \\
\hline Klebsiella pneumoniae & & & & & & $\mathrm{V}$ & $\mathrm{V}$ & V \\
\hline Providencia rettgeri & & & & & & $\mathrm{V}$ & & \\
\hline Acinetobacter baumannii & & & & & & & $\mathrm{V}$ & V \\
\hline Morganella morganii & & & & V & & & & \\
\hline Staphylococcus epidermis & & & & $\mathrm{V}$ & & & & \\
\hline
\end{tabular}

CIP, ciprofloxacin; SAM, ampicillin-sulbactam; PIP, piperacillin; CRO, ceftriaxone; VA, vancomycin; CEF, cefazolin; TEC, teicoplanin; CAZ, ceftazidime; VAN, vancomycin; LVX, levofloxacin; MPM, meropenem.

GM, gentamicin; AN, amikacin; FEP, cefepime; TZP, piperacillin-tazobactam; IPM, imipenem; TGC, tigecycline; S, sensitive; R, resistant; I, intermediate.

MRSA, methicillin-resistant Staphylococcus aureus.

neighboring slots, along with the transfer tube and the corresponding suspension tube. Calculations were performed on raw data and compared to thresholds to determine the reactions for each test.

\section{Statistical Analysis}

The difference between wound-healing patients and no-woundhealing patients in terms of blood sugar values (at 6 a.m., 11 a.m., 5 p.m., and 9 p.m.) were evaluated using the Student $t$ test for continuous variables. All statistical analyses were conducted using the statistical package SPSS for Windows version 17.0 (SPSS, Inc., Chicago, IL, USA). A $p$ value $<0.05$ was considered statistically significant.

\section{Results}

Nine cases of $A$. faecalis skin and soft tissue infection were seen during the study period; One case had a surgical wound infection. Eight cases had diabetic foot ulcer infections, including 5 male and 3 female patients. The mean age was 54.6 years (range $41-85$ years). All patients had other comorbidities. All foot ulcer lesions were of chronic duration (>14 days; range 14 days to 18 months). Two patients had no history of prior IV antibiotics used within 3 months for their chronic ulcer lesion. All wound cultures displayed polymicrobial infection. The antibiotics sensitivity test showed the presence of extensive drug-resistant (XDR) A. faecalis beginning in 2019. Two patients did not receive adequate antibiotic therapy. Case 4 was treated with oxacillin and cefmetazole, which do not treat $A$. faecalis. Case 7 was treated with ceftazidime and vancomycin, which do not treat A. faecalis and Acinetobacter baumannii. All patients needed surgical intervention for their chronic ulcer lesions. The wound did not heal in 3 patients.

Tables 1-3 show the clinical characteristics, pharmacological therapy for blood sugar, admission $\mathrm{HbAlc}$ values, 1-week average blood sugar values, treatment outcomes, 
Table 3. The 1-week average of blood sugar values and pharmacological therapy in 8 cases of diabetic foot ulcer with Alcaligenes faecalis infection

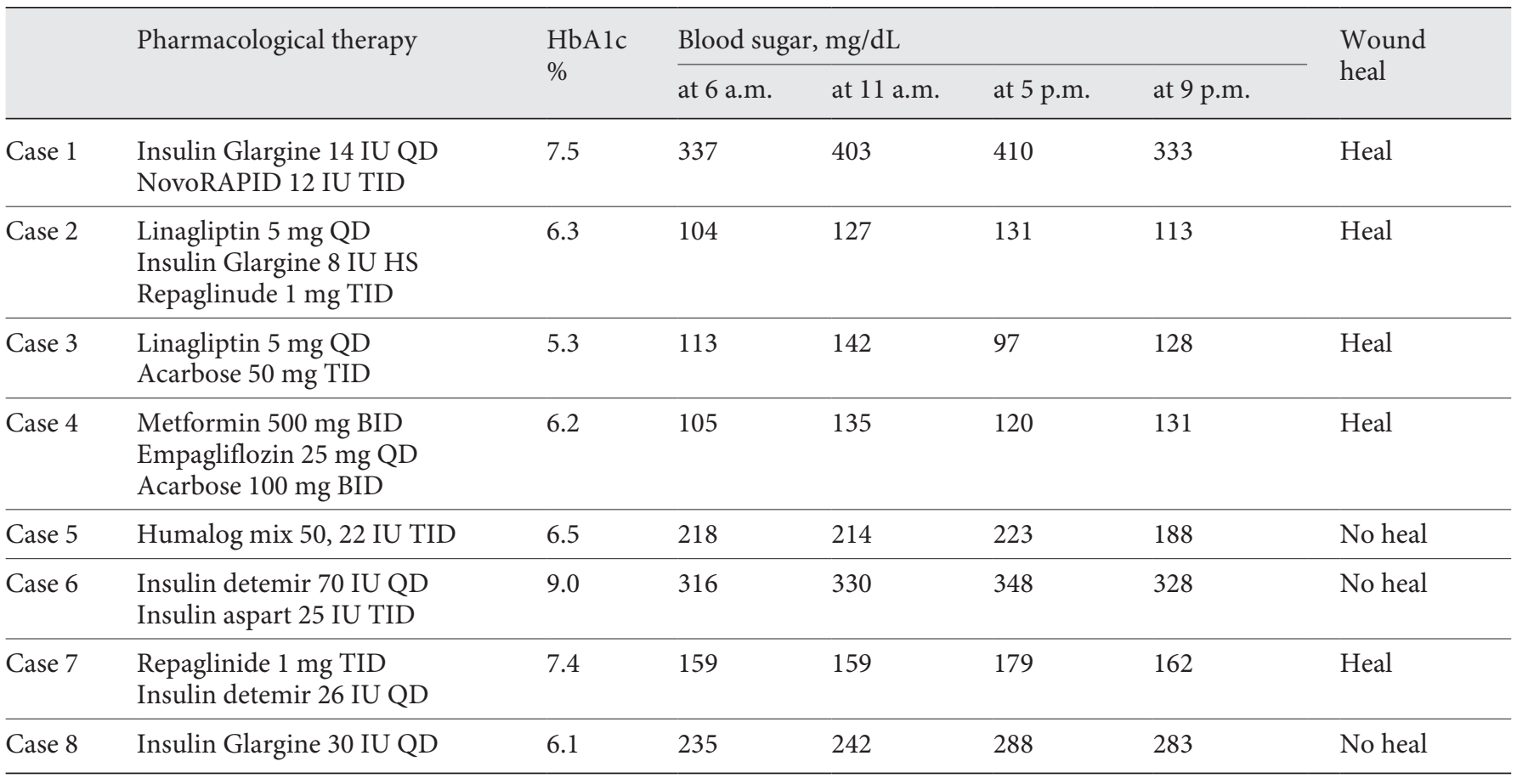

and bacteriology of the 8 patients with diabetic foot ulcer with $A$. faecalis infection. The average blood sugar values at 6 a.m. were significantly different between wound-healing patients and no-wound-healing patients $(p=0.008)$. The average blood sugar values at 11 a.m. were not significantly different between wound-healing patients and no-wound-healing patients $(p=0.108)$. The average blood sugar values at 5 p.m. were significantly different between wound-healing patients and no-wound-healing patients $(p=0.028)$. The average blood sugar values at 9 p.m. were significantly different between wound-healing patients and no-wound-healing patients ( $p=0.009)$.

\section{Discussion}

The literature shows 12 cases of skin and soft tissue $A$. faecalis infection, including 4 cases of toe web infection [23], 1 case of dog bite cellulitis [24], 2 cases of surgical wound infection [17], 1 case of superinfection in the foot tinea pedis [17], and 4 cases of diabetic foot ulcer infection $[13,15,17]$. Other studies and our case series indicate that diabetic foot ulcer accounts for $57.1 \%$ of all $A$. faecalis infections in skin and soft tissue. In 1997, Bizet and Bizet [13] reported the case of a 64-year-old male with insulin-dependent diabetes and left foot ulcer with $A$. faecalis infection. The patient was treated with IV amoxicillin-clavulanic acid for 10 days [13]. In 2014, Tena et al. [17] reported 2 cases of diabetic foot ulcer with A. faecalis infection, all with polymicrobial infection. All strains of A. faecalis were susceptible to amoxicillin-clavulanic acid. One case was a cured foot ulcer, and the other case was a recurrence of foot ulcer [17]. In 2019, Chua et al. [15] reported the case of a 63-year-old female with a diabetic foot ulcer with extended spectrum $\beta$-lactamase (ESBL)producing $A$. faecalis infection. They suggested that imipenem and meropenem may be considered as agents of choice for an ESBL-producing $A$. faecalis infection [15].

In our series, all cases had an ulcer lesion of chronic duration ( $>14$ days), and all had mixed infection with other bacteria. The most common additional pathogens were Enterococcus, Klebsiella pneumoniae, Proteus vulgaris, and A. baumannii. Gram-negative bacilli account for $71.4 \%$ of all mixed infection pathogens. In 2017, the first case with $A$. faecalis resistant to gentamicin and ciprofloxacin was reported. Unfortunately, diabetic foot ulcer with XDR A. faecalis infection emerged in 2019, and IV tigecycline or colistin was needed to treat it. There is no literature on XDR A. faecalis diabetic foot ulcer infection, but 2 cases of XDR A. faecalis pneumonia and 1 case 
of pandrug-resistant $A$. faecalis bacteremia have been reported [19, 20, 25]. In 2018, Junejo et al. [19] reported on a 73 -year-old male with $A$. faecalis pneumonia. The sensitivity analysis of the sputum culture showed that the isolated $A$. faecalis was resistant to anti-pseudomonas penicillins, carbapenems, aminoglycosides, and quinolones. The patient was treated with polymyxin B and became hemodynamically stable [19]. In 2017, Agarwal et al. [20] reported on a 32-year-old man with Dengue hemorrhagic fever and pneumonia. Bronchoalveolar lavage fluid culture isolated A. faecalis, and the antibiotic sensitivity test showed sensitivity to colistin and tigecycline only. Colistin and meropenem were given, but the patient later succumbed to his illness [20]. In our Cases 7 and 8, the antibiotic sensitivity test was the same as in the cases of Agarwal et al. and Junejo et al.

In 2019, Hasan et al. [25] reported on a 60-year-old female with pandrug-resistant $A$. faecalis who was treated with double-dose tigecycline and had a successful treatment outcome. It was terrible that pandrug-resistant an $A$. faecalis pathogen had been found. We emphasize that $A$. faecalis has emerged as a pathogen resistant to commonly used antibiotics such as anti-pseudomonas penicillins, carbapenems, aminoglycosides, and quinolones. It is too early to know the pathogenesis of emerging multidrug-resistant A. faecalis. The medical community urgently needs microbiologists to explore the pathogenesis of emerging multidrug-resistant $A$. faecalis, so as to control this pathogen.

Although Cases 4 and 7 did not receive adequate IV antibiotics to treat the $A$. faecalis infection, they received surgical intervention and wound care. Their diabetic foot ulcer infections were healing. Their diabetic foot ulcers were local infections that could be cured by adequate debridement and appropriate wound care. This result stresses that wound care and surgical interventions are important in treating diabetic foot ulcer infections. In 3 cases, the diabetic foot ulcer did not heal. Case 5 had wound infection complicated by septic shock. This patient had to undergo right lower leg below the knee amputation to save her life. Case 6 had peripheral artery disease (PAD) and chronic kidney disease as comorbidities. The duration of his diabetic ulcer lesion was 12 months. The patient received IV antibiotics with ceftazidime and tigecycline therapy, as well as debridement, but the leg ulcer lesion still failed to heal. Case 8 had PAD, chronic kidney disease, bed-ridden hypoxic encephalopathy, and prolonged mechanical ventilation as comorbidities. The duration of his diabetic ulcer lesion was 18 months. The ulcer lesion showed infection with XDR A. faecalis. The patient was treated with IV colistin and wound debridement, but the ulcer lesion still failed to heal. Two of the 3 cases that failed to heal had PAD comorbidity and the 2 cases did not undergo any revascularization procedures. PAD may be an influential factor of poor wound healing in diabetic foot ulcer. The study by Brechow et al. found that the severity of PAD significantly influences the ability of diabetic foot ulcer to heal [26]. Weck et al. reported that poor wound healing is often aggravated by PAD as comorbidity [27].

Many factors can interfere with diabetic foot ulcer healing, such as wound infection control, blood glucose control, immune function, nutrition, etc. In Christman et al.'s study, it was suggested that glycemia may be an important biomarker in predicting wound-healing rate in diabetic patients [28]. Markuson et al.'s study showed that healing took significantly longer for individuals with lower admission $\mathrm{HbA} 1 \mathrm{c}$ values compared with those with higher levels [29]. Our report of diabetic foot ulcer with A. faecalis infection consists of only 8 cases, and we could not draw any clinical conclusions by admission HbAlc. We collected our 8 patients' blood sugar level 4 times a day for a week and found that optimizing glucose control is an influencing factor of diabetic foot ulcer healing.

The infection of diabetic foot ulcers may contribute to delayed healing. Optimal management of diabetic foot infections can reduce the incidence of infection-related morbidities and the incidence of limb amputation. Antibiotic therapy is necessary for all infected wounds. Definitive therapy should be based on both the culture results and susceptibility data. It is important to remember that all patients will need appropriate wound care, and most will need rapid surgical intervention.

\section{Limitations}

Our case series report of diabetic foot ulcer with $A$. faecalis infection is small and therefore can provide only minimal clinical experience. Additional reports of diabetic foot ulcer with $A$. faecalis infection will add to the knowledge of how to treat this multi-resistant pathogen infection.

\section{Conclusion}

Diabetic foot ulcer with $A$. faecalis infection is rarely reported in the literature. XDR $A$. faecalis has emerged recently in our hospital. Diabetic foot ulcer with $A$. faecalis infection is common in those with an ulcer lesion of chronic duration (>14 days), and the infections are commonly polymicrobial. Adequate antibiotic treatment, appropriate wound care, and surgical intervention are all crucial for healing. 


\section{Statement of Ethics}

The project was approved by Buddhist Dalin Tzu Chi General Hospital's research Ethics Committee (approved IRB No.: B10802024). Written informed consents were obtained from the patients for publication of this report.

\section{Conflict of Interest Statement}

The author declares that they have no competing interests.

\section{Author Contributions}

This is a single-author paper. C.H. designed the study, collected the data, analyzed the data, wrote the manuscript, and reviewed the manuscript.

\section{Availability of Data and Materials}

The datasets used and/or analyzed during the current study are available from the corresponding author upon reasonable request.

\section{Funding Sources}

This study received no funding.

\section{References}

1 Lavery LA, Armstrong DG, Wunderlich RP, Mohler MJ, Wendel CS, Lipsky BA. Risk factors for foot infections in individuals with diabetes. Diabetes Care. 2006 Jun;29(6):1288-93.

2 Zhang P, Lu J, Jing Y, Tang S, Zhu D, Bi Y. Global epidemiology of diabetic foot ulceration: a systematic review and meta-analysis †. Ann Med. 2017 Mar;49(2):106-16.

3 Singh N, Armstrong DG, Lipsky BA. Preventing foot ulcers in patients with diabetes. JAMA. 2005 Jan;293(2):217-28.

4 Prompers L, Schaper N, Apelqvist J, Edmonds M, Jude E, Mauricio D, et al. Prediction of outcome in individuals with diabetic foot ulcers: focus on the differences between individuals with and without peripheral arterial disease. The EURODIALE Study. Diabetologia. 2008 May;51(5):747-55

5 Pathare NA, Bal A, Talvalkar GV, Antani DU. Diabetic foot infections: a study of microorganisms associated with the different Wagner grades. Indian J Pathol Microbiol. 1998 Oct; 41(4):437-41.

6 Sapico FL, Witte JL, Canawati HN, Montgomerie JZ, Bessman AN. The infected foot of the diabetic patient: quantitative microbiology and analysis of clinical features. Rev Infect Dis. 1984 Mar-Apr;6 Suppl 1:S171-6.

7 Wheat LJ, Allen SD, Henry M, Kernek CB, Siders JA, Kuebler T, et al. Diabetic foot infections. Bacteriologic analysis. Arch Intern Med. 1986 Oct;146(10):1935-40.

8 Urbancic-Rovan V, Gubina M. Bacteria in superficial diabetic foot ulcers. Diabet Med. 2000 Nov; 17(11):814-5.

9 Ge Y, MacDonald D, Hait H, Lipsky B, Zasloff M, Holroyd K. Microbiological profile of infected diabetic foot ulcers. Diabet Med. 2002 Dec;19(12):1032-4.

10 Mordi RM, Yusuf EO, Onemu SO, et al. The prevalence of Alcaligenes faecalis in bacteremia, meningitis and wound sepsis in a tertiary health care institution in western part of $\mathrm{Ni}$ geria. Int J Biotechnol. 2013;2:123-9.
11 Mordi RM, Burke ME, Odjadjare EE, Enabulele SA, Umeh OJ. Prevalence of urinary tract infections among pregnant women in university of Benin teaching hospital (UBTH) Benin City, Nigeria. J Asian Scient Research. 2015;5(4):198-204.

12 Siddiqui N, Kirmani S, Khan F, Kaushal N, Shukla IK, Khan HM, et al. Risk Factors of Candida blood Stream Infections in a Tertiary Care Hospital. Int J Curr Microbio App Sci. 2015 Special Issue-1:157-62.

13 Bizet J, Bizet C. Strains of Alcaligenes faecalis from clinical material. J Infect. 1997 Sep; 35(2):167-9.

14 Cole AC, Marshall C. Infective endocarditis due to Bact. Faecalis alcaligenes. BMJ. 1952 Oct;2(4789):867.

15 Chua KH, Puah SM, Puthucheary SD. First report of extended-spectrum $\beta$-lactamases TEM-116 and OXA-10 in clinical isolates of alcaligenes species from Kuala Lumpur, Malaysia. J Jpn J Infect Dis. 2019 Jul;72(4):266-9.

16 Kaliaperumal S, Srinivasan R, Gupta A, Parija SC. Postoperative endophthalmitis due to an unusual pathogen: alcaligenes faecalis. Eye (Lond). 2006 Aug;20(8):968-9.

17 Tena D, Fernández C, Lago MR. Alcaligenes faecalis: an unusual cause of skin and soft tissue infection. Jpn J Infect Dis. 2015;68(2): $128-30$.

18 Kavuncuoglu F, Unal A, Oguzhan N, Tokgoz B, Oymak O, Utas C. First reported case of Alcaligenes faecalis peritonitis. Perit Dial Int. 2010 Jan-Feb;30(1):118-9.

19 Junejo SZ, Tuli S, Trandafirescu T. A rare case of pneumonia caused by Alcaligenes Faecalis bacteria. Am J Respir Crit Care Med. 2018; 197:A3602.

20 Agarwal A, Sharma S, Bhargava V, Bhargava V, Agarwal M, Airun M. First reported case of Alcaligenes faecalis isolated from bronchoalveolar lavage in a patient with dengue hemorrhagic fever. J Assoc Chest Physicians. 2017; 5(1):51-5.
21 Lavery LA, Armstrong DG, Harkless LB. Classification of diabetic foot wounds. J Foot Ankle Surg. 1996 Nov-Dec;35(6):528-31.

22 Lipsky BA, Silverman MH, Joseph WS. A proposed new classification of skin and soft tissue infections modeled on the subset of diabetic foot infection. Open Forum Infect Dis. 2016 Dec;4(1):ofw255.

23 Aste N, Atzori L, Zucca M, Pau M, Biggio P. Gram-negative bacterial toe web infection: a survey of 123 cases from the district of $\mathrm{Ca}$ gliari, Italy. J Am Acad Dermatol. 2001 Oct 45(4):537-41.

24 Chu AS, Harkness J. Alcaligenes faecalis cellulitis after a dog bite: case report and literature review. Pediatr Emerg Care. 2017 Jul 33(7):497-8.

25 Hasan MJ, Nizhu LN, Rabbani R. Bloodstream infection with pandrug-resistant Alcaligenes faecalis treated with double-dose of tigecycline. IDCases. 2019 Jul;18:e00600.

26 Brechow A, Slesaczeck T, Münch D, Nanning T, Paetzold H, Schwanebeck U, et al. Improving major amputation rates in the multicomplex diabetic foot patient: focus on the severity of peripheral arterial disease. Ther Adv Endocrinol Metab. 2013 Jun;4(3):83-94.

27 Weck M, Slesaczeck T, Rietzsch H, Münch D, Nanning T, Paetzold $\mathrm{H}$, et al. Noninvasive management of the diabetic foot with critical limb ischemia: current options and future perspectives. Ther Adv Endocrinol Metab. 2011 Dec;2(6):247-55

28 Christman AL, Selvin E, Margolis DJ, Lazarus GS, Garza LA. Hemoglobin A1c predicts healing rate in diabetic wounds. J Invest Dermatol. 2011 Oct;131(10):2121-7.

29 Markuson M, Hanson D, Anderson J, Langemo D, Hunter S, Thompson P, et al. The relationship between hemoglobin $\mathrm{A}(1 \mathrm{c})$ values and healing time for lower extremity ulcers in individuals with diabetes. Adv Skin Wound Care. 2009 Aug;22(8):365-72. 